APRIL

NSDI (Usenix Symposium on Networked Systems Design and Implementation)

April 11-13, 2007

Cambridge, Massachusetts

http://www.usenix.org/events/nsdi07/

Gelato ICE (Itanium Conference and Expo)

April 15-18, 2007

San Jose, California

http://www.ice.gelato.org/

Web 2.0 Expo

April 15-18, 2007

San Francisco, California

http://www.web2expo.com/

SaaScon (Software as a

Service Conference)

April 17-18, 2007

Santa Clara, California

http://www.saascon.com/live/48/

MySQL Conference and Expo

April 23-26, 2007

Santa Clara, California

http://www.mysqlconf.com/

\section{PostgreSQL Bootcamp}

April 23-27, 2007

Atlanta, Georgia

http://bignerdranch.com/classes/

postgresql.shtml

\section{MAY}

HotOS (Workshop on Hot Topics in Operating Systems)

May 7-9, 2007

San Diego, California

http://www.usenix.org/events/

hotos07/

STAREAST (Software Testing

Analysis and Review)
May 14-18, 2007

Orlando, Florida

http: //www.sqe.com/stareast/

Communications Developer

Conference

May 14-17, 2007

Santa Clara, California

http://www.tmcnet.com/

voipdeveloper/

\section{RailsConf}

May 17-20, 2007

Portland, Oregon

http://conferences.oreillynet.com/ rails/

OSBC (Open Source Business

Conference)

May 22-23 2007

San Francisco, California

http://www.osbc.com/live/13/

O'Reilly Where 2.0 Conference

May 29-30, 2007

San Jose, California

http: //conferences.oreillynet.

com/where2007/

JUNE

Tech $\cdot$ Ed

June 4-8, 2007

Orlando, Florida

http://www.microsoft.com/events/

teched2007/default.mspx

\section{IBM Rational Software}

Development Conference

June 10-14, 2006

Orlando, Florida

http://www-306.ibm.com/software/

rational/events/rsdc2007/

Workshop on Experimental Com-

puter Science (part of ACM Federated Computing Research Conference)

\section{To announce \\ an event, E-MAIL}

QUEUE-ED@ACM.ORG OR

FAX +1-212-944-1318

June 13-14, 2007

San Diego, California

http://www.cs.huji.ac.il/

$\sim$ feit/exp/

Usenix Annual

Technical Conference

June 17-22, 2007

Santa Clara, California

http: //www.usenix.org/events/

usenix07/

\section{Better Software Conference}

and Expo

June 18-21, 2007

Las Vegas, Nevada

http://www.sqe.com/

bettersoftwareconf/

JULY

$\mathrm{CIO}$ and CSO Business

Continuity Forum

July 17-18, 2007

New York, New York

http: //public.cxo.com//conferences//

index. html?conferencelD $=6$

Networkers at Cisco Live

July 22-26 2007

Anaheim, California

http://www.cisco.com/web/learning/

le21/le34/networkers/nw07

OSCON (O'Reilly Open Source

Convention)

July 23-27, 2007

Portland, Oregon

http://conferences.oreillynet.com/ os2007/ 